\title{
Y a-t-il un mouvement paysan en Afrique noire ?
}

\section{Dominique Gentil, Marie-Rose Mercoiret}

\section{Citer ce document / Cite this document :}

Gentil Dominique, Mercoiret Marie-Rose. Y a-t-il un mouvement paysan en Afrique noire ?. In: Tiers-Monde, tome 32, $n^{\circ} 128$, 1991. Politiques agraires et dynamismes paysans : de nouvelles orientations ? pp. 867-886;

doi : https://doi.org/10.3406/tiers.1991.4633

https://www.persee.fr/doc/tiers_0040-7356_1991_num_32_128_4633

Fichier pdf généré le 29/03/2018 


\title{
Y A-T-IL UN MOUVEMENT PAYSAN EN AFRIQUE NOIRE?
}

\author{
par Dominique GentiL*, Marie-Rose MerCoIRET**
}

La crise des Etats en Afrique noire, la domination idéologique du libéralisme et ses implications pratiques dans les Programmes d'Ajustement structurel font ressurgir un intérêt pour la ( société civile ), les dynamiques endogènes, les entrepreneurs privés et les organisations paysannes. Dans l'espérance d'une nouvelle répartition des rôles et du transfert de certaines fonctions actuellement détenues par l'Etat, l'existence ou non d'un mouvement paysan revêt donc une réelle importance.

Après une discussion sur les critères de définition d'un mouvement paysan, nous essayerons de tracer les grandes lignes d'évolution des organisations paysannes au cours de ces trente dernières années. Quelques exemples d'organisations significatives devraient permettre d'illustrer la situation présente et d'identifier les conditions d'émergence et d'évolution d'un tel mouvement.

\section{L'AJUSTEMENT DE LA RÉPONSE ET DES CRITÈRES}

La réponse à la question $Y$ a-t-il un mouvement paysan en Afrique noire? dépend des critères que l'on retient pour définir ce qu'est " un mouvement paysan ». La réponse pourra être positive, négative ou nuancée selon que les critères seront inspirés de modèles occidentaux ou (c exotiques ", du mouvement paysan français mêlant la cogestion économique, le syndicalisme et l'influence politique, ou des révolutions paysannes mexicaines, chinoises ou vietnamiennes ${ }^{1}$.

* IRAm, Paris.

** DSA/CIRAD, Montpellier.

1. Cf. E. Wolf, Les guerres paysannes du vingtième siècle, Paris, Maspero, 1971.

Revue Tiers Monde, t. XXXII, n॰ 128, Octobre-Decembre 1991 
D'une manière sans doute arbitraire et discutable, nous avons retenu un ensemble de cinq critères pour tenter d'apprécier l'existence de mouvements paysans.

Une autonomie intellectuelle et financière. - Des emprunts à des modèles étrangers, des appuis financiers extérieurs ne disqualifient pas, bien sûr, un mouvement paysan, mais il ne peut être considéré comme tel que s'il a une réelle aptitude à définir son champ d'activité, ses modes d'organisation, à prendre ses propres décisions, s'il dispose d'un minimum d'autofinancement qui lui donne une marge de manœuvre par rapport à l'Etat ou à ses autres bailleurs de fonds.

Des objectifs conscients et explicites. - Les membres doivent s'y reconnaître et y adhérer même s'ils participent diversement à leur définition... Ces objectifs expriment alors un projet d'avenir, une " vision du monde ", une ( idéologie ), qui soudent les membres.

Des rapports significatifs avec l'Etat et/ou le reste de la société civile. Ce qui distingue un ( mouvement ) des dynamiques purement endogènes du secteur dit informel, c'est qu'il apparaît au grand jour; il négocie des alliances, entre en concurrence ou en conflit avec d'autres acteurs sociaux, des institutions étatiques, des entreprises, des Eglises, des partis, d'autres mouvements sociaux.

Une taille ou un poids économique/politique " suffisant". - Il n'y a pas de critère objectif de cette suffisance, mais il est clair que quelques groupements paysans isolés ne font pas un mouvement et que des organisations qui restent marginales économiquement ou qui ne pèsent pas dans les rapports de force politiques ne peuvent être considérées comme un mouvement.

Une organisation interne déjà établie. - Il peut y avoir plusieurs centaines d'unités de base, ayant un poids économique significatif et des objectifs communs qui restent cependant des entités séparées, ou se rencontrant seulement d'une manière occasionnelle ${ }^{2}$.

Qui dit mouvement dit circulation de l'information, échanges, capacité de décisions collectives, de respect des décisions prises, de contrôle... en un mot capacité de "( coopération 》 des diverses unités de base.

Pour vérifier la pertinence de ces critères, il est intéressant de faire définir par les organisations paysannes elles-mêmes ce qu'elles entendent par ( mouvement ). Un livre récent de $P$. Pradervand ${ }^{3}$ reproduit quelques citations de leaders paysans qui permettent d'illustrer leur conception.

2. Cf. infra, par exemple le cas des Associations villageoises de la CMDT.

3. Pierre Pradervand, Une Afrique en marche, Paris, Plon, 1989. 
Pour Abdoulaye Diop, fondateur de l'amicale du Walo ${ }^{4}$ sur le fleuve Sénégal, le mouvement paysan est déjà en marche : ‘ J'ai beaucoup d'espoir pour le mouvement paysan, car nous avons aujourd'hui des paysans qui pensent leur propre développement. Dans moins de quinze ans, grâce aux paysans, on réussira à atteindre ce "moins d'Etat" que vise le Président de la République, parce que tous ces milliards dépensés par les sociétés d'intervention... eh bien, on pourra en dépenser dix fois moins avec les mouvements paysans parce qu'ils se sentent responsables de leur propre avenir... Maintenant, les paysans disent : nous sommes capables de tout faire. Nous pouvons penser par nous-mêmes, être responsables de nous-mêmes. ')

Par ailleurs, Mamadou Cissoko, un des fondateurs et des animateurs de l'Entente de Koupentoum, parle, lui, de pouvoir paysan : " Je suis très optimiste pour l'avenir. Je vois une montée au pouvoir des leaders paysans, mais de façon pacifique. ) Une des premières étapes semble être la participation aux instances locales du pouvoir ${ }^{5}$.

Ces deux leaders d'organisations parlent explicitement de mouvement ou de pouvoir paysan dans un avenir proche. Ils soulignent la capacité de penser par soi-même (autonomie intellectuelle), d'avoir sa propre conception du développement (idéologie propre) et de créer de nouveaux rapports économico-politiques avec l'Etat.

Cette tendance s'inscrit dans une évolution sur une trentaine d'années des organisations paysannes, avec leur autonomie croissante vis-à-vis de l'Etat.

DES COOPÉRATIVES ÉTATIQUES AUX ORGANISATIONS « ENDOGÈNES 》

L'histoire des organisations paysannes en Afrique a déjà fait l'objet de nombreuses analyses : rapports d' (c experts ), thèses et diplômes (notamment autour du Collège coopératif de Paris) ou livres plus synthétiques ${ }^{6}$. Il est notamment possible d'établir une typologie relativement simple avec trois formes essentielles, qui se sont en partie succédé, même s'il y a de nombreux chevauchements ou coexistences.

4. Cf. infra, les organisations fédératives sénégalaises.

5. A. Bamba Thialene, les deux tiers des membres de la Communauté rurale sont membres de l'Entente de Koupentoum (cf. Pradervand).

6. Par exemple : M.-R. Mercoiret, L'émergence des dynamiques locales. Une réponse au désengagement de l'Etat, Montpellier, septembre 1990 ; D. Gentil, Les mouvements coopératifs en Afrique de l'Ouest. Intervention de l'Etat ou organisations paysannes, Paris, L'Harmattan, 1986 ; D. Gentil, A. Le Gentil, A. Marty, J.-B. Spinat, L'appui aux organisations paysannes en Afrique, Paris, Caisse centrale de coopération économique, juin 1990. 
- Au moment des Indépendances, beaucoup d'espoirs avaient été mis dans les coopératives, qui étaient considérées, dans la version du « socialisme africain ") (Sénégal, Niger, Tanzanie...), comme un des moyens de réconcilier les " solidarités traditionnelles et la modernisation technique » ou, dans la vision marxisante (Guinée Conakry, Mali, Bénin, Congo, Angola, Mozambique, Ethiopie...), comme un moyen d'introduire le socialisme dans les campagnes (passage de la petite production parcellaire à l'exploitation collective, et ( révolution scientifique et technique )). Des appareils spécifiques de promotion avaient été créés à cet effet, des législations promulguées (très souvent avec l'appui du BIT), des campagnes de sensibilisation, des programmes de formation et des appuis divers mis en place.

D'une manière générale, et bien qu'il y ait eu dans certains cas et à certaines périodes des exceptions intéressantes où les coopérateurs ont vraiment participé à la définition des modes d'organisation et avaient un pouvoir réel de décision - même si celui-ci était souvent partiel ${ }^{7}$-, ces coopératives étatiques sont restées étrangères à la paysannerie. L'initiative, le mode d'organisation inspiré du juridisme occidental, les prises de décision, accaparées par les fonctionnaires et/ou les notables ruraux, les formes de contrôle inadaptées ont fait de ces organisations des " coopératives sans coopérateurs ", des instruments de l'Etat et non pas l'affaire des paysans. Certaines de leurs fonctions économiques ont pu être appréciées par les membres (commercialisation plus honnête, approvisionnement en biens de consommation à bas prix, disponibilité de crédit - souvent mal remboursé -, infrastructures de stockage...), mais leur champ d'activité restait essentiellement limité à ce qui intéressait l'Etat, c'est-à-dire la promotion des cultures d'exportation (arachide, huile de palme, café, cacao...). De plus, de nombreux détournements ou la non-prise en compte des contraintes économiques ont débouché progressivement sur une asphyxie puis une faillite économique.

Ces coopératives d'initiative étatique ne pouvaient donc pas être le support d'un mouvement paysan. Elles ont eu pour résultat, le plus souvent, de créer un effet répulsif par rapport au terme même de coopératives. Dans la majorité des cas, elles ont disparu ou ne subsistent qu'à l'état de coquilles vides. Certaines conservent cependant une réelle efficacité économique (l'UCCAO ${ }^{8}$ au Cameroun, par exemple) mais la participation des membres y est limitée.

7. Début des indépendances au Sénégal avec un rôle actif de l'animation, Sabon Tsari au Niger (1966-1970), etc.

8. uCCAO : Union des Cooperatives Caféières de l'Ouest. 
- Beaucoup plus vivantes sont les organisations mixtes, prenant le nom d'associations ou de groupements, qui se sont créées le plus souvent comme des compromis ou des convergences d'intérêt entre des paysans et des sociétés, offices ou projets de développement pour prendre en charge des fonctions précises et éviter le formalisme, les pesanteurs bureaucratiques ou les tentatives prédatrices des divers appareils officiels de promotion coopérative. Les exemples les plus significatifs, dont certains seront développés plus loin, concernent les associations cotonnières et les associations ou groupements situés sur les aménagements hydroagricoles. Dans le cas du Mali Sud, le point de départ en 1973-1974 semble être la revendication par les paysans d'effectuer eux-mêmes la pesée du coton pour mettre fin aux controverses auxquelles elle donnait lieu et la volonté d'une nouvelle équipe de la CMDT (Compagnie malienne de Développement des Textiles) de promouvoir des organisations paysannes pour transférer aux paysans, contre une rémunération limitée, certaines fonctions économiques (approvisionnement, crédit, commercialisation) ${ }^{9}$.

Dans ce type d'organisation, les constantes sont la prise en charge de fonctions économiques bien précises (gestion du réseau hydraulique, de batteuses à riz, commercialisation, approvisionnement...), l'existence d'intérêts réciproques, une base villageoise et souvent le refus du modèle officiel des coopératives.

Ce modèle est largement répandu dans de nombreux pays. Par exemple, les associations ou groupements cotonniers se retrouvent dans une dizaine de pays et se rencontrent dans près de 10000 villages, mais on ne peut guère encore parler à cet égard de mouvement paysan ${ }^{10}$.

- Les organisations d'initiative non étatique se sont multipliées depuis une vingtaine d'années, avec le soutien actif des organisations non gouvernementales (ONG) d'appui au gouvernement.

Sous des appellations diverses, avec ou sans statuts juridiques (associations, groupements, ententes, groupements d'intérêt économique, coopératives...), il existe dans certains pays des milliers d'organisations de ce type. A travers la multiplicité des formes, il faut donc tenter de comprendre les mécanismes de création de ces nouvelles structures et leurs caractéristiques spécifiques pour savoir s'il s'agit enfin de l'amorce d'un authentique mouvement paysan, créant ses propres formes d'organisation et disposant d'une large autonomie vis-à-vis de l'Etat ou, en fait, de structures marginales dispersées, sans avenir réel prévisible.

9. Il existe plusieurs versions de ce point de départ, preuve qu'il est la résultante de plusieurs facteurs. Certains auteurs insistent aussi sur la continuité des formes « traditionnelles », les tons avec les nouvelles associations villageoises.

10. Cf. infra, la section sur les Associations villageoises au Mali-Sud et à l'Office du Niger. 
L'origine de ces organisations peut servir de critère de différenciation; on peut ainsi distinguer les organisations suscitées par les $O N G$ et les organisations reliées au mouvement associatif, d'initiative locale, "( endogène ).

Les premières, très nombreuses notamment dans la plupart des pays du Sahel, sont très diverses tant par leurs objectifs, leurs activités et leurs performances que par leur capacité d'évolution, de mûrissement.

A côté de formes d'organisation ( communautaires ), qui s'appuient souvent sur des ressorts socioculturels (solidarités traditionnelles) et qui ont des objectifs globaux multisectoriels, on trouve des groupements plus spécialisés; ainsi certains groupements sont centrés sur une activité de production (maraîchage, artisanat, petit élevage), sur une fonction économique en amont ou en aval de la production (comités de gestion des banques de céréales, de magasins villageois, caisses d'épargne et de crédit, etc.); d'autres ne concernent qu'une catégorie sociale (jeunes, femmes) ou professionnelle (artisans, pêcheurs, etc.).

Souvent nées autour de micro-réalisations, les organisations paysannes suscitées par des oNG évoluent de façon très variable : certains groupements restent très dépendants de leurs appuis extérieurs; les ( après-projets ), quand les financements tarissent, sont parfois douloureux et « la prise en charge " par les intéressés eux-mêmes ne se fait pas sans mal. Des évolutions plus positives existent heureusement et certaines de ces organisations sont capables de reproduire les premières actions, de les élargir, de devenir multisectorielles, etc.

Les organisations paysannes liées au mouvement associatif résultent, quant à elles, d'une initiative locale et sont autonomes par rapport à l'Etat. C'est au Sénégal et au Burkina Faso qu'elles sont les plus nombreuses et les plus dynamiques et qu'elles revêtent la forme d' "( organisations paysannes intervillageoises $)^{11}$.

Elles se définissent en règle générale des objectifs globaux (amélioration des conditions d'existence, autosuffisance alimentaire); le projet général, d'ordre " social ), est souvent mobilisateur et la difficulté centrale est de le traduire en actions concrètes. Toutes les organisations de ce type n'y parviennent pas; celles qui obtiennent les meilleurs résultats sont bien sûr celles qui à partir d'un projet global, à moyen et long terme, sont capables de mobiliser les compétences et les moyens internes et externes pour mettre au point et diffuser les innovations (techniques, économiques, en gestion, etc.) indispensables pour atteindre leurs objectifs ${ }^{\mathbf{1 2}}$.

11. Cf. infra, les organisations paysannes fédératives au Sénégal et les groupements « Naam 》.

12. L'Entente de Koumpentoum, l'ASECCAW, CADEF, l'AJAC, etc., au Sénégal, les groupements Naam au Burkina Faso par exemple, cf. infra. ASECCAW : Association socio-économique, 
Vis-à-vis d'éventuels partenaires extérieurs, les organisations “ associatives " font de la reconnaissance de leur autonomie un préalable mais des différences notables existent entre organisations; pour réduire les dépendances qui peuvent s'instaurer, certaines diversifient leurs partenaires techniques et leurs sources de financement; cela leur permet d'obtenir les indispensables appuis techniques et financiers tout en leur ménageant des marges de manœuvre et de négociation importantes...

Jusqu'à ces dernières années, les activités des organisations non étatiques présentaient trois caractéristiques essentielles, les différenciant des organisations d'initiative étatique :

- une très forte tendance à la multifonctionnalité;

- l'imbrication étroite entre l'économique et le social;

- des activités économiques dans des secteurs relativement " marginaux》 ou délaissés par les organismes officiels de développement.

Une exception importante cependant mais qui confirme la règle : la forte expansion à partir de 1970 des coopératives d'épargne et de crédit ${ }^{13}$. En fait, compte tenu des résultats très décevants de la collecte de l'épargne par le circuit officiel (banques et caisses nationales d'épargne), l'Etat, au départ, considérait qu'il s'agissait là d'un secteur marginal et difficile qu'il laissa occuper par les ONG ou les aides extérieures spécialisées.

Les choses évoluent et on note un nombre croissant d'organisations non étatiques pénétrant dans les circuits stratégiques (par exemple périmètres rizicoles, crédit agricole ou commercialisation de l'arachide).

D'une manière générale, ces trois tendances (secteurs ( marginaux ), multifonctionnalité, imbrication de l'économique et du social) constituent une très grande richesse mais peuvent aussi limiter le développement futur des organisations paysannes.

Les organisations paysannes non étatiques sont trop diverses pour qu'on puisse parler à leur propos ( de mouvement paysan ). A côté des organisations paysannes intervillageoises du Sénégal et du Burkina Faso qui témoignent de l'émergence d'un mouvement paysan, on trouve en effet des milliers de groupements atomisés, sans projets et sans moyens propres, flottant au gré de la conjoncture... L'enjeu est sans doute pour les premières de pouvoir entraîner ces groupements, de savoir élargir avec eux la base du mouve-

sportive et culturelle des Agriculteurs du Walo. CADEF : Comité d'action pour le développement de Fogny. AJAC : Association des Jeunes Agriculteurs de Casamance.

13. Cf. par exemple : D. Gentil et Y. Fournier, Coopératives d'épargne et de crédit et voies alternatives au financement du développement rural en Afrique « francophone », Notes et Etudes, Paris, Caisse centrale de Coopération économique, novembre 1988. 
ment dont elles sont l'avant-garde. Les rapprochements qui s'opèrent (FONGS, CORD par exemple) ${ }^{14}$ semblent montrer que cela est possible même si la voie n'est pas aisée.

ESQUisSe D'Un TABLEAU DES MOUVEMENTS PAYSANS POTENTIELS

Cette description typologique et ce bref rappel historique permettent de dresser à grands traits le panorama des organisations paysannes. Il nous faut maintenant, avec un brin d'arbitraire et sans doute quelques oublis, passer rapidement au crible de nos cinq critères (autonomie intellectuelle et financière, vision du monde commune, rapports avec l'extérieur, poids économico-politique, degré d'organisation) les expériences les plus significatives, avec un accent particulier sur le Sénégal où la situation est la plus riche.

\section{A / Les organisations paysannes fédératives au Sénégal}

Le Sénégal offre une large gamme d'organisations paysannes; nous ne retiendrons ici que des organisations fédératives, de deuxième voire de troisième niveau, car elles seules peuvent annoncer l'émergence d'un mouvement paysan.

Il existe en milieu rural un mouvement associatif, autonome par rapport à l'Etat, qui s'est développé à partir du début des années 70 et qui a pris de l'ampleur au cours de la dernière décennie. Il s'est développé dans les régions " périphériques 》 (Fleuve, Sénégal oriental, Casamance) et a gagné progressivement le bassin arachidier.

Les organisations pionnières sont désormais célèbres : l'ASESCAW dans la région de Saint-Louis, l'AJAC et l'Entente de Diouloulou en Casamance, l'Entente de Bamba-Thialene au Sénégal oriental, etc. Fédérant des groupements, des « foyers de jeunes », plus récemment des GIE (groupements d'intérêt économique) au sein d'organisations paysannes intervillageoises appelées parfois (" associations villageoises de développement ", elles ont fait école (CADEF dans le département de Bignona, UFuLAL ${ }^{15}$ et AJAEDO $^{16}$ à Oussouye, AISB $^{17}$ et " Paysans organisés de Bakel » dans le

14. FONGS : Fédération des ONG du Sénégal. CORD : Coordination des Organisations professionnelles rurales du Département de Bignona.

15. UFULAL : nom casamançais.

16. AJAEDo : Association de Jeunes Agriculteurs et Eleveurs du Département Oussouye.

17. AISB : Association intervillageoise de Sinthiou-Boubou. 
département de Bakel, ARAF ${ }^{18}$ dans la région de Fatick, ADAK ${ }^{19}$ dans celle de Kaolak, etc.), et il y en a aujourd'hui des dizaines regroupant des dizaines voire peut-être quelques centaines de milliers d'adhérents.

Ces organisations paysannes intervillageoises se sont souvent créées avec des statuts d'association (type loi 1901) et leur reconnaissance n'a pas toujours été aisée. Elles revendiquent leur autonomie vis-à-vis des intervenants extérieurs (services étatiques et para-étatiques) avec lesquels elles ont parfois eu dans le passé des relations tendues (notamment dans la région du Fleuve) surtout lorsqu'elles avaient choisi une stratégie différente de celle de la Société de Développement. La nouvelle politique agricole leur est très favorable et les collaborations avec l'Administration se multiplient, sans cependant qu'elles renoncent à leurs partenaires de toujours que sont les ong.

On trouve le plus souvent à l'origine des Organisations paysannes intervillageoises un leader ayant un degré d'information et de formation supérieur à la moyenne. Celui-ci a su cependant la plupart du temps favoriser l'émergence de nouveaux leaders paysans et le pouvoir généralement partagé.

Elles ont démarré toutes très lentement avec un travail approfondi de réflexion collective, parfois long. Le point de départ semble avoir presque toujours été un autodiagnostic de la situation vécue et le désir de « s'en sortir en comptant sur ses propres forces 》). La sécheresse et les conséquences sur la situation alimentaire ont souvent servi de ressorts à cette démarche mais parfois aussi la volonté d'affirmer un projet de développement différent de celui qui était proposé par les structures étatiques de développement.

Elles fédèrent des groupements de base dont elles coordonnent et impulsent les actions et auxquels elles apportent divers appuis (formation, prestations de services, crédit, financements, etc.). Ces organisations de base sont diverses : des foyers et de plus en plus des GIE à l'ASASCAW, des groupements de producteurs au niveau infra-villageois (Kabiline, AJAC), des comités villageois (CADEF, BambaThialene), etc. Des structures intermédiaires existent encore entre les organisations de base et la coordination centrale (zones, unions, comités, etc.).

Leurs programmes ont en règle générale connu un élargissement progressif : si les objectifs généraux sont restés à peu près inchangés (autosuffisance alimentaire, amélioration des conditions de vie), elles ont débuté le plus souvent par des actions à caractère social (santé, petite hydraulique, etc.), de diversification (maraîchage, petit élevage, arboriculture) et se sont attaquées progressivement aux problèmes plus complexes liés à l'agriculture (pluviale ou irriguée) et à l'élevage : production de semences, approvisionnement, commercialisation, transformation des produits, crédit, etc.

Elles bénéficient toutes de l'appui d'oNG, du Nord mais aussi du " Nord-Sud 》 (( Six-S ») $)^{20}$ qui apportent des soutiens financiers importants et un appui métho-

18. ARAF : Association régionale des Agriculteurs de Fatick.

19. ADAK : Association départementale des Agriculteurs de Kaolack.

20. « Se Servir de la Saison Sèche en Savane et au Sahel », cf. infra. 
dologique. Conscientes des dépendances qui peuvent se créer, beaucoup diversifient leurs partenaires et se donnent les moyens de rester maîtresses de leur projet (formation en particulier).

Des écarts de compétence importants existent parfois entre les responsables des organisations et leur base. La nécessité de saisir des opportunités, notamment dans la période actuelle du fait du désengagement de l'Etat, pousse en effet certains “ exécutifs » à avancer très vite, à prendre des décisions seuls, etc. Des instances de contrôle et de régulation existent dans toutes les organisations (conseils d'administration, comités de coordination, etc.), mais il n'est pas certain que la décentralisation optimale soit respectée dans tous les $\operatorname{cas}^{21}$.

Certaines organisations ont une base territoriale "continue »: c'est le cas de Bamba-Thialène (Entente de Koumpentoum) et du CADEF par exemple et sans doute aussi de l'AISB, etc. Cela constitue un bon point de départ pour la définition de stratégies de développement local multisectorielles. D'autres sont plutôt des fédérations d'organisations infravillageoises dispersées géographiquement même si elles restent dans un espace relativement homogène : l'ASESCAW dans le Delta, l'AJAC en Casamance, relèvent de cette catégorie. Elles orientent alors plus leurs actions vers l'organisation de prestations de service aux groupements.

La plupart d'entre elles tendent à se fédérer afin d'accroître leur autonomie interne (échanges d'expériences et de produits), et leur pouvoir de négociation avec l'extérieur. Ces fédérations de troisième niveau peuvent être départementales (coordination des organisations rurales du département de Bignona-CORD), régionales (Union régionale de l'AJAC par exemple en Casamance, ASESCAW, Association régionale des agriculteurs de Fatick), ou interrégionales (InterEntente de Bamba-Thialène). Nombreuses sont en outre les organisations de ce type qui ont adhéré à la Fédération des ong du Sénégal (FONGS) dont le siège est à Thiès.

La FONGS admet comme membres des organisations intervillageoises (Ententes de Koumpentoum, Diouloulou, etc.), des unions de groupements (Maisons familiales rurales, etc.), des associations régionales (AJAC, ASESCAW, ARAF, ADAK, etc.).

Les actions menées par la FoNGS sont nombreuses ; on peut citer :

- la mise en place en 1985 d'un système de formation qui fonctionne selon une programmation établie de façon concertée : formation à la gestion, à la planification, formation des animateurs d'association, alphabétisation. L'objectif est toujours la formation de formateurs capables de démultiplier les acquis auprès de leurs organisations ;

- la mise en place d'un système de sécurité alimentaire permettant des échanges entre associations excédentaires et associations déficitaires ;

- la mise en place en 1987 d'un système d'épargne et de crédit en relation avec la Caisse nationale de Crédit agricole (CNCAS). L'épargne collectée et une subvention de 156 millions de CFA ont permis à la FONGS de négocier avec

21. Ces phénomènes ne sont pas bien sûr spécifiques aux organisations sénégalaises, et on les retrouve aussi dans les pays du Nord ! 
la CNCAS et de permettre à des organisations non éligibles au crédit (faute de statut juridique) d'y avoir accès avec la garantie de la FONGS. L'ASESCAW, Kabiline, Bamba-Thialène ont entre autres bénéficié de cette opération qui ne portait dans un premier temps que sur les crédits de campagne. La FONGS souhaite devenir membre du Conseil d'Administration de la CNCAS afin d'avoir un rôle dans la définition des orientations de cet organisme.

La FONGS est devenue au niveau national « le partenaire paysan » incontournable ; bien qu'elle ne puisse pas prétendre à la représentation des paysans organisés sénégalais dans leur totalité, elle regroupe cependant les organisations associatives les plus anciennes et son audience ne cesse de croître.

Les organisations fédératives liées à la mouvance associative semblent pour la plupart en train d'émerger en tant que mouvement paysan, même si elles sont à des stades divers de maturation et d'efficacité.

Toutes affirment dans les faits leur autonomie intellectuelle parfois avec une assurance qui peut surprendre certains appareils de développement peu habitués encore à négocier avec les paysans. Toutes sont porteuses d'un " projet d'avenir " explicite et les plus solides ne font pas mystère de leur finalité (négocier une insertion plus favorable de la paysannerie dans la société globale). Toutes entretiennent des relations négociées de tous ordres avec les autres acteurs du développement (Etat, ONG, secteur privé, etc.) et s'appliquent, en faisant valoir leur base, à se faire reconnaître comme partenaires à part entière; elles tirent de ces relations avec l'extérieur une légitimation et une efficacité accrues, qui renforcent leur crédibilité interne. L'appui des ONG les met en position favorable pour négocier avec les services de l'Etat et la diversification des partenaires techniques et financiers étrangers (oNG du Nord et du Sud) limite des dépendances (nées de l'exclusivité de l'appui) qui ont pu exister parfois au départ... Toutes ont conscience de la nécessité d'une organisation à des échelons supérieurs (département, région, etc.) pour échanger des expériences et des produits, mettre en place des services collectifs et acquérir un pouvoir de négociation avec l'environnement public et privé; des structures diverses se mettent en place pour y parvenir. Par leur taille les organisations paysannes de type associatif ne peuvent pas prétendre encore à la représentativité des paysans sénégalais; le mouvement existe, se développe et se renforce mais ne touche pas encore la majorité des paysans. En revanche, le poids politique des organisations est relativement important à la fois par l'absence d'autres formes d'organisations crédibles pour les paysans et par la stratégie qu'elles ont adoptée d'investir les collectivités locales décentralisées.

Leur poids économique est encore sans doute moins important que leur poids politique; cependant, ces organisations ne se réduisent pas à des 
( groupes de pression 》 et ne fonctionnent pas comme tels. Elles ont des initiatives nombreuses : la création de services à la production en amont et en aval surtout, mais aussi façons culturales, etc.), promotions d'entreprises (GIE par exemple), renforcement de la sécurité alimentaire, etc.; ces initiatives améliorent les conditions de production (même s'il y a des errements dus à des choix techniques plus ou moins pertinents) et accroissent le poids économique des organisations. Le choix d'intervenir " au sommet ) (entrée au Conseil d'Administration de la CNCA par exemple) est justifié lui aussi par cette préoccupation.

\section{B / Autres expériences africaines}

Les groupements Naam sont nés dans la province du Yatenga au Burkina Faso en $1967^{22}$. C'est à notre connaissance l'organisation paysanne qui valorise le plus explicitement les ressorts culturels de la société rurale pour favoriser l'émergence de formes d'organisation devant affronter les défis “ modernes »; la continuité entre le Kombi-Naam, structure sociale traditionnelle mossi ( ( Pouvoir des Jeunes 》) et le groupement Naam est sans cesse rappelée par les animateurs qui définissent ce dernier comme la version adaptée de l'association traditionnelle, respectueuse « des valeurs et coutumes héritées (solidarité, savoir et savoir-faire), mais intégrant les valeurs modernes (technologie, écriture, etc.), qui fondent le développement économique et social ).

Les groupements Naam ont débordé le cadre du Yatenga pour toucher 17 provinces; il en existe, d'après les responsables, environ 3000 au Burkina Faso regroupant 350000 membres originaires de 1200 villages.

Le groupement Naam de base compte au moins 50 personnes et peut se constituer au sein du village ou du quartier; les « unions » sont le premier échelon de structuration fédérative (il y en a 63) et il existe, au niveau national, une Fédération des Unions des Groupements Naam (FUGN).

Les groupements Naam ont un projet qui s'exprime dans ( une philosophie ) (" développer sans abîmer 》), " un but 》 (construire une nouvelle société fondée sur une " démocratie qualitative », ce qui exclut le recours à des statuts juridicoadministratifs importés par exemple...), un objectif général, l'autoresponsabilisation pour atteindre " les objectifs secondaires " que sont l'autosuffisance et la sécurité alimentaire, la lutte contre la désertification, etc.

Les groupements Naam ont vu leur efficacité accrue par la création, en 1976, de l'oNG Six-S ${ }^{23}$, dont l'exécutif est à Ouahigouya et que B. L. Ouédraogo définit comme un " Naam international ». L'association Six-S vise en effet à apporter un appui aux initiatives des paysans organisés, " sans les écraser ».

22. Bernard Lédéa Ouédraogo, Entraide paysanne et développement au Burkina Faso, Paris, Ed. L'Harmattan, 1990.

23. Savoir se Servir de la Saison Sèche en Savane et au Sahel. 
Disposant de moyens importants fournis par un consortium de financement (CEBEMo, IDA suisse, Misereor, CCFD, Action de Carême suisse), Six-S apporte une aide technique et financière aux Groupements Naam.

L'action de Six-S a dépassé les frontières du Burkina Faso et diverses organisations paysannes dans sept autres pays d'Afrique de l'Ouest bénéficient de son appui ${ }^{24}$. Son Conseil d'Administration et son Assemblée générale sont composés de délégués issus des associations paysannes.

On peut considérer que les groupements Naam - Six-S constituent un mouvement paysan en émergence : l'autonomie intellectuelle de l'organisation est avérée depuis longtemps et la formule adoptée avec la création de Six-S lui permet de fonctionner avec une autonomie financière sur le moyen terme. Le projet d'avenir des groupements Naam est sans doute l'un des plus explicites qui soient au plan global, idéologique; en revanche, et au Yatenga en particulier, du fait des contraintes agro-écologiques très fortes qui pèsent sur la production, il peut sembler un peu difficile que l'autoresponsabilisation soit suffisante pour trouver des réponses aux graves problèmes qui se posent aux producteurs; sans doute la création récente du Centre international de Recherche sur les technologies paysannes à Ouahigouya est-elle un moyen de progresser dans ce sens.

Les groupements Naam ont su négocier des alliances avec divers partenaires extérieurs en particulier, la structuration interne de l'organisation est établie aux échelons départemental et national et son articulation avec Six-S lui confère un rôle au plan international.

\section{Les Coopératives d'Epargne et Crédit (Coopec)}

Sous des noms divers, les Crédits Unions ou Coopec ont déjà une longue histoire en Afrique, particulièrement dans les pays anglophones (notamment Ghana). Dans la zone francophone les Coopec apparaissent surtout dans les années 1970 (Centre Sud Cameroun, Casamance, Burkina Faso, Zaïre, Côte-d'Ivoire, Bénin, Togo, Rwanda). Elles sont, sauf rares exceptions, à initiative non étatique mais avec presque toujours un soutien plus ou moins extérieur (missions catholiques ou protestantes, centres de formation, crédit mutuel français ou suisse, mouvement Desjardins du Québec...). Une nouvelle vague de création marque la fin des années 1980 (Mali, Burundi, Sénégal, Congo, Guinée). Elle n'est pas étrangère à la dégradation des systèmes centralisés de distribution de crédit (Caisses ou Banques nationales de Crédit agricole) et à l'intérêt des bailleurs de fonds envers les voies alternatives en matière d'épargne et de crédit. 
Leur poids économique s'accroît rapidement : les expériences anciennes comme l'Ouest-Cameroun, le Rwanda, la plupart des pays de l'Afrique de l'Est, le Bénin, le Togo ou le Burkina dépassent chacune 20 millions de francs français d'épargne et distribuent environ la moitié de celle-ci en crédit le plus souvent correctement remboursé. Les Coopec s'organisent presque toujours en deux ou trois étages (unions locales, régionales ou nationales).

Il y a donc un poids économique réel dans un secteur, le financement, qui est bien sûr décisif. Les Coopec et leurs Unions sont reconnues par l'Etat mais défendent en général jalousement leur autonomie à son égard. Elles passent des accords avec les banques nationales pour placer leurs disponibilités financières, intéressent les Banques centrales, négocient avec les bailleurs de fonds.

Mais en règle générale, ce type d'organisation reste très inspiré d'un modèle extérieur (européen, québécois ou américain), qui est expliqué aux membres, grâce à la sensibilisation ou à la formation. Il est rarement une création originale ou l'objet d'une véritable appropriation sociale, notamment en ce qui concerne les modalités de décision ou de contrôle. Les critères de viabilité financière restent déterminants. Les décisions les plus importantes ont tendance à revenir aux techniciens des Unions et à leurs conseillers et il y a souvent coupure entre les caisses de base et leurs administrations paysannes et les Unions. De plus les Coopec se veulent avant tout une structure financière spécialisée dans la sécurisation de l'épargne et la distribution de crédit et ne cherchent pas à diffuser, en liaison avec d'autres organisations, une conception originale du ( développement ).

\section{Les Associations villageoises à Mali Sud et à l'Office du Niger (Mali)}

Les Associations villageoises à Mali Sud sont le résultat d'un compromis d'intérêts entre les paysans et la CMDT (cf. supra).

Après plus de quinze ans d'expérience, elles concernent maintenant plus de 1000 villages. Elles assurent la collecte primaire de la quasi-totalité du coton, distribuent les intrants agricoles, sélectionnent les emprunteurs et récupèrent les crédits et, grâce à leurs excédents, investissent dans de nombreux équipements sociaux (puits, cases de santé, maternités rurales, centres d'alphabétisation...) et économiques (mini minoteries, ateliers de forgerons, biogaz...). Une des originalités et sans doute une des conditions de réussite a été l'effort soutenu en matière d'alphabétisation intensive (plus de 5000 auditeurs formés tous les ans), qui a permis l'émergence d'un auto-encadrement paysan, non seulement pour les fonctions économiques ou sociales déjà citées mais aussi dans le domaine technique (vulgarisation, vaccination des animaux, collecte de statistiques...). Celui-ci reçoit 
des indemnités, prélevées sur la marge de commercialisation ( 2 à $3 \mathrm{~F} \mathrm{CFA} / \mathrm{kg}$ de coton) qui restent modestes mais qui, dans de nombreux cas, permettent aux jeunes de rester au village plutôt que de partir en exode. Ainsi un certain compromis social s'établit entre anciens et jeunes, les premiers gardant l'essentiel du pouvoir ou au moins son apparence, mais sachant que les jeunes alphabétisés sont indispensables pour le bon fonctionnement des associations et qu'il faut donc accepter une partie des " compensations ) demandées si l'on veut les maintenir au village.

Depuis quelques années (1983), une structure intermédiaire, la ZAER (Zone d'Animation de l'Expansion rurale), réunit 3 à 15 villages, pour des fonctions qui dépassent le cadre villageois. Ces ZAER ont un statut qui reste cependant ambigu : organisations paysannes intervillageoises, dernier niveau de l'encadrement de la CMDT ou lieu de confrontation entre les logiques paysannes et les logiques d'intervention para-étatiques.

De plus, depuis 1988, des caisses intervillageoises d'épargne et crédit, les Kafo Jiginew ${ }^{25}$, apportent une dimension financière supplémentaire et regroupent déjà plus de 5000 membres.

U n'y a donc pas véritablement de mouvement paysan : la dépendance vis-à-vis de la CMDT ou de l'extérieur reste grande même si elle est en partie voulue (la CMDT est un partenaire fiable, implanté depuis des dizaines d'années, qui sert de protecteur vis-à-vis des " tentations 》 de l'administration), l'échelon intervillageois reste limité, l'élaboration d'un projet explicite reste inexistante, mais il y a un poids économique, une extension géographique, une dynamique d'auto-encadrement et de réflexion paysanne relativement impressionnante, une prise de conscience encore timide de la force potentielle, qui laissent prévoir la possibilité d'organisations mieux structurées $^{26}$.

A l'Office du Niger, vaste aménagement hydro-agricole de 50000 ha, ancien fleuron de la colonisation (créé en 1932), les associations villageoises sont beaucoup plus récentes (1984-1985). Une de leurs premières activités a été la gestion de petites batteuses à riz (deux à trois par village), permettant de limiter leur dépendance vis-à-vis des grandes batteuses de l'Office et de constituer une première source de revenus. D'autres fonctions ont suivi comme le crédit, le stockage, l'approvisionnement en intrants et en bœufs de labour, la commercialisation...

Si l'essentiel des activités se déroule au niveau du village qui se confond avec l'AV, il existe aussi des regroupements occasionnels de 5 à 10 AV pour des fonctions spécifiques : lancer un appel d'offres pour obtenir de l'engrais, négocier avec une coopérative d'éleveurs ou un gros commerçant pour obtenir des bœufs de labour. Plus récemment (1990-1991), des représentants de 26 Associations siègent avec

25. Soutenus par la Fondation du Crédit coopératif et diverses ONG.

26. Les derniers événements politiques au Mali (1991) ont montré cependant la capacité des associations à présenter et à défendre une plate-forme réaliste de revendications. 
un rôle actif à un Comité paritaire de Gestion d'un Fonds de redevance, qui décide et contrôle l'utilisation des différents travaux hydrauliques. En 1991 les AV commencent à avoir la charge de la gestion du terroir et donc aussi de l'épineux problème d'attribution/exclusion des parcelles irriguées.

Par rapport à un schéma dominant très directif (et pour certaines époques c'est un euphémisme) ${ }^{27}$, la création des Associations villageoises, avec leurs multiples fonctions économiques, ouvre de nouveaux rapports beaucoup plus contractuels avec l'office du Niger. La situation est cependant loin d'être encore irréversible (les terres continuent d'appartenir à l'Etat) et les associations, même si elles se rencontrent et par moments coopèrent, restent avant tout centrées sur les affaires de leur propre village.

DES CONDITIONS D'ÉMERGENCE, D'ÉVOLUTION

ET DE REPRODUCTIBILITÉ D'UN MOUVEMENT PAYSAN ${ }^{28}$

Le recul historique est insuffisant pour se prononcer sur l'existence d'un véritable mouvement paysan en Afrique noire; si, les premières étapes semblent avoir déjà été franchies dans certains pays, ceux-ci restent encore l'exception.

A partir des études de cas précédentes, il est possible d'apporter quelques éclaircissements sur la conjonction de facteurs favorables à l'émergence d'un mouvement paysan.

- Un environnement politique favorable qui permette l'autonomie des organisations. Plusieurs cas de figures sont cependant possibles : il peut s'agir d'Etats " démocratiques ) qui ont déjà une société civile développée

27. Voir, par exemple, pour la période coloniale : Amidu Magassa, Papa commandant a jeté un grand filet devant nous, Maspero, 1978. Mais la situation en 1980 n'était guère plus enviable.

28. L'existence de leaders locaux est un facteur favorable, voire nécessaire, à l'apparition d'organisations non étatiques durables. Dans le mouvement associatif, les leaders paysans sont souvent de fortes personnalités, à cheval entre deux mondes : véritables paysans mais ayant voyagé, ayant su tisser des liens familiaux ou amicaux avec des groupes extérieurs au monde paysan, fonctionnaires renonçant à leur poste, travailleurs migrants (parfois ex-responsables syndicaux en Europe)... Des biographies complètes et comparatives restent à faire. On peut noter cependant à la fois un enracinement réel avec le milieu paysan ainsi qu'une capacité a servir d'intermédiaire avec le milieu extérieur, soit national, soit international.

Il existe aussi de nombreux groupements où l'initiative est partie de groupes sociaux particuliers, notamment les jeunes et les migrants, ayant vécu ensemble certaines expériences (les classes d'âge, l'école, le travail en exode, la ville...) et ne pouvant accepter la continuité de la vie économique (faible revenu, travail fatigant) et sociale (pouvoir des anciens) du village. Dans certains cas, mais encore relativement rares et seulement dans certaines régions, il s'agit de groupes de femmes recherchant, par des activités spécifiques et acceptables par les hommes (maraîchage, artisanat, transformation des produits, petit commerce...), des revenus complémentaires. 
(multipartisme, syndicats indépendants, presse libre...), d'Etats " déliquescents " qui acceptent toute initiative qui ne remet pas en cause directement leurs privilèges, ou d'Etats " sous influence ", qui doivent accepter, pour obtenir des financements et au moins payer leurs fonctionnaires, le démantèlement des sociétés d'Etat et Offices, la libéralisation, la privatisation, et donc ( le transfert de responsabilités 》 à des organisations paysannes.

- Des enjeux clairement identifiés par les membres, dont ils ont conscience qu'ils dépassent le cadre local. La force et la faiblesse des organisations paysannes résident certainement dans leur enracinement au niveau villageois. Là les problèmes sont ressentis, les normes sociales de décision et de contrôle sont disponibles, la transparence est simplifiée. Mais la tentation est de rester à ce niveau, de chercher par exemple une solution particulière à l'écoulement de son riz sans essayer de se grouper pour faire front aux pratiques des commerçants ou d'organiser son propre circuit de vente jusqu'à la capitale. Les activités doivent donc présenter des possibilités d'économies d'échelle ressenties par les membres pour inciter à dépasser le cadre villageois.

- Une capacité d'organisation interne. L'alchimie qui aboutit à une organisation efficace et durable est toujours un peu mystérieuse. On en connaît la plupart des ingrédients (existence et qualité des leaders mais aussi leur contrôle par la base, capacité de réflexion collective, de circulation de l'information, de gestion financière et économique, de transparence dans la gestion, de formes de contrôle comptable et social...), mais les combinaisons peuvent être diverses et les fruits plus ou moins savoureux. Les déviations possibles sont aussi bien répertoriées et bien présentées ${ }^{29}$ : leaders charismatiques qui accaparent le pouvoir et les privilèges, et qui se coupent de leur base, faux leaders qui cherchent surtout à capter une rente extérieure et qui font fonctionner les organisations pour leurs propres intérêts économiques ou politiques, faible participation des membres dont les problèmes ou les avis ne sont pas pris en compte, comptabilité obscure et jamais présentée d'une manière compréhensible aux membres...

- Des activités et des espaces économiques maîtrisables. Les organisations paysannes débutent par des opérations relativement simples (la collecte primaire des produits et non pas l'exportation, le crédit local et non pas la gestion des banques...), qui se situent à un niveau local ou microrégional. Les responsables peuvent ainsi facilement se rencontrer et restent très proches de leurs membres. Ils connaissent leurs terroirs et leurs problèmes, et essaient de valoriser au mieux leur potentiel.

29. Ces déviations ne sont pas, bien évidemment, une spécificité africaine ! 
- La capacité de nouer des alliances internes (au niveau national) ou externes (ONG, Eglises, bailleurs de fonds...). Il est rare qu'une organisation puisse se développer uniquement par ses propres forces. Des complicités ou des alliances sont presque toujours nécessaires pour obtenir des moyens financiers complémentaires, mais peut-être surtout pour obtenir des informations, échanger des expériences, quelquefois obtenir un débouché pour ses productions, acquérir un minimum de sécurité par rapport au gouvernement par la reconnaissance internationale ou l'appui, au moins implicite, de leaders politiques nationaux ou de contre-pouvoirs comme les Eglises.

L'évolution d'un mouvement paysan appelle quelques remarques. L'histoire des mouvements paysans en France, en Europe et aussi en Amérique latine montre qu'ils ne se construisent pas selon une " progression linéaire ", et que les évolutions tourmentées, conflictuelles, en " dents de scie » sont bien plus la règle que les montées en puissance " harmonieuses ". Il semble qu'il en sera de même en Afrique et on peut déjà voir les signes avant-coureurs de ces accouchements difficiles... Un mouvement paysan résulte en règle générale de dynamiques et de stratégies multiples.

Ainsi, dans le delta du fleuve Sénégal le rôle historique de l'asescaw est incontestable; elle a ouvert la voie, créé un espace d'initiatives pour les paysans et... a suscité des émules qui peuvent devenir, sur certains aspects, des concurrents vis-à-vis des groupements de base. A une moindre échelle, le CADEF a ouvert une voie dans l'arrondissement de Sindian qu'une organisation plus récente - YAMAKAY - a suivie et cela se traduit de fait par des concurrences auprès des adhérents potentiels. Si l'on comprend sans peine que ces concurrences soient exaspérantes pour les responsables des organisations, elles peuvent cependant être considérées comme une ( saine émulation 》 dès lors qu'elles ne visent pas à la surenchère démagogique ou au dénigrement, qui ne pourraient profiter qu'aux acteurs sociaux extérieurs au monde paysan. L'enjeu semble être alors pour ces initiatives de se « fédérer 》 pour accroître le pouvoir de négociation vis-à-vis de l'extérieur sans chercher à tout prix et d'entrée de jeu des “ fusions».

De la même manière, les organisations paysannes traversent « des crises de croissance " qui peuvent se traduire ici et là par des scissions : conflits de pouvoir entre " leaders historiques », divergences idéologiques ou opérationnelles, conflits entre leaders historiques et nouveaux leaders suscités par l'organisation, etc.

Ces phénomènes “ font désordre » pour les technocrates qui rêvent d'organigrammes clairs, ils sont difficiles à vivre pour les responsables, et ils ne coïncident pas avec les phantasmes de certains idéologues tiersmondistes. Loin d'inquiéter, ces phénomènes devraient plutôt rassurer ceux 
qui espèrent l'émergence d'un véritable mouvement paysan en Afrique; ils témoignent de l'existence de réelles dynamiques économiques, sociales et politiques, et prouvent que ces organisations sont vivantes...

Toutes les organisations, tous les leaders et responsables ne choisissent pas la même stratégie... Certains se méfieront des « fédérations 》 de troisième ou quatrième niveau et préféreront investir leur énergie dans le renforcement de dynamiques locales de développement; d'autres au contraire (ou simultanément) jugeront indispensable de faire émerger au plan national une représentation de la paysannerie... C'est ainsi qu'au Sénégal il nous semble fort heureux que la FONGS soit reconnue comme interlocuteur paysan par les pouvoirs publics et par leurs grands bailleurs de fonds; même si certaines organisations se tiennent en dehors, c'est un grand progrès, nous semble-t-il, que ( son message ) émerge au plan politique et que ces paysans d'un type nouveau deviennent des partenaires ayant à se prononcer sur des choix nationaux qui les concernent. Son action devrait accroître la marge de mancuvre des organisations locales et rendre l'environnement plus favorable aux organisations qui ont choisi de se renforcer à des échelles géographiques inférieures.

La recherche d'efficacité, notamment économique et politique, pousse à la spécialisation de certaines fonctions et de certains hommes... C'est là un processus inéluctable qu'ont connu les autres mouvements paysans. Ce faisant, la distance entre les adhérents de base et les responsables des structures mises en place s'accroît, et on arrive à des coupures entre le sommet et la base. Il est vraisemblable qu'il en sera de même en Afrique et que les mêmes causes produiront les mêmes effets... cela est parfois déjà perceptible dans certaines organisations. On ne peut qu'espérer que, en Afrique comme ailleurs, les organisations de base seront suffisamment vivantes pour entretenir une " contestation ) interne; l'absence de contestation est en effet un signe de mauvaise santé; elle révèle souvent soit une base sans projet, (" amorphe ), soit une bureaucratisation suffisamment pesante pour étouffer les dynamiques créatrices.

CONCLUSION

Même si l'étude est loin d'être exhaustive, les expériences analysées ne représentent pas encore, sauf peut-être au Sénégal, un véritable mouvement paysan ayant une taille suffisante, une organisation efficace, un projet d'avenir le rendant capable de peser dans les rapports de force de la vie nationale. Les organisations paysannes se limitent pour le moment à des 
activités économiques. Les aspects idéologiques ${ }^{30}$ ou culturels restent peu développés. Compte tenu du contexte politique (en général parti unique ou parti dominant, syndicat peu autonome vis-à-vis du gouvernement), elles ne se sont pas lancées, directement ou indirectement, dans le champ politique ou syndical, même si certains de leurs leaders ont une politique d'infiltration au niveau local. Même dans le cas d'un multipartisme naissant (Bénin, Niger et peut-être bientôt Mali), elles n'ont pas été capables de faire entrer les problèmes des paysans, qui représentent cependant la majorité de la population, dans le champ des enjeux politiques. Il n'y a pas de propositions structurées pour des politiques agricoles alternatives et les débats restent encore cantonnés au niveau urbain.

Mais le rôle économique des organisations paysannes s'accroît et se diversifie, et les activités ne se cantonnent plus aux secteurs marginaux. Il n'y a pas encore de mouvement paysan dans la plupart des cas, mais des amorces et des potentialités existent.

Il est certainement possible en cette période de doute et de focalisation sur la crise des Etats de parler de " révolution silencieuse ") : le secteur dit informel (bien qu'il obéisse à des règles précises) se développe dans l'artisanat, la transformation, le commerce, les services personnels ${ }^{31}$ ou l'épargnecrédit (tontines, banques ambulantes, caisses de solidarité...). Des milliers de groupements, d'hommes, de femmes, de jeunes mènent des activités économiques et sociales. Enfin, des organisations intervillageoises créent les prémices d'un véritable mouvement paysan. De plus en plus, les paysans renoncent à des stratégies d' " escapade ", de " non-capture ") (cf. Hyden) pour essayer des relations contractuelles avec l'extérieur.

Il faut cependant être très conscient que cette amorce de mouvement paysan n'existe pas dans tous les pays et reste très fragile. Des dissensions internes, des répressions étatiques, des changements de conjoncture économique, des abandons de supports financiers ou techniques extérieurs peuvent très bien faire disparaître, au moins temporairement, ces dynamiques et ces potentialités.

30. La “ théorisation », la réflexion sur l'avenir et les difficultés de ces mouvements paysans restent peu élaborées, à part quelques variations sur le thème de la tradition et de la modernité, surtout si on les compare à la floraison idéologique des indépendances.

31. Cf. par exemple Abdou Touré, Les petits métiers à Abidjan. L'imagination au secours de la conjoncture, Paris, Karthala, 1985. 